\title{
Melanoma primario del esófago tratado con esofagectomía. Casos clínicos
}

\author{
JEAN M. BUTTE ${ }^{1}$, ÁLVARO VISSCHER ${ }^{a}$, HERNÁN DE LA FUENTE ${ }^{1}$, \\ MANUEL MENESES ${ }^{2}$, ANA MARÍA CARRASCO ${ }^{2}$, \\ HORACIO AMARAL ${ }^{3}$, ENRIQUE WAUGH ${ }^{1}$
}

\section{Esophageal melanoma. Report of two cases}

Esophageal melanomas correspond to 0.1 to $0.2 \%$ of esophageal tumors. We report two patients with the disease. The first patient is a 51 year-old woman presenting with dysphagia and weight loss. An upper gastrointestinal endoscopy showed a polypoid ulcerated lesion in the middle third of the esophagus. The pathological study of the biopsy disclosed a malignant melanoma. The patient was subjected to an esophagectomy with a satisfactory postoperative evolution. Four months later, liver metastases were detected and the patient died eleven months after the operation. The second patient is a 59 year-old male that consulted by dysphagia. An endoscopy showed a pigmented esophageal lesion whose pathological diagnosis was a malignant melanoma. The patient was subjected to an esophagectomy and sixteen months after surgery there was no evidence of relapse.

(Rev Med Chile 2010; 138: 77-81).

Key words: Esophageal neoplasms; Melanoma.

'Servicio de Cirugía Digestiva Oncológica. ${ }^{2}$ Anatomía Patológica.

${ }^{3}$ Medicina Nuclear. Instituto Oncológico Fundación Arturo López Pérez (FALP) Santiago, Chile.

Interno de Medicina, Universidad de Los Andes.

Recibido el 3 de abril de 2009 aceptado el 10 de noviembre de 2009.

Correspondencia a : Dr. Enrique Waugh Fundación Arturo López Pérez Rancagua 878

Santiago, Chile Teléfono: 56-2-4457247

Fax: 56-2-4218597

E-mail: ewaugh@mi.cl.
L os melanomas primarios del esófago son infrecuentes, representan entre 0,1 y $0,2 \%$ de las neoplasias esofágicas y $0,5 \%$ de los melanomas viscerales ${ }^{1}$. Se estima una incidencia de 0,0036 casos por cada millón de personas por año ${ }^{2}$.

La mayoría de los pacientes consultan inicialmente por disfagia lógica y el diagnóstico se puede sospechar al momento de la endoscopia digestiva alta (EDA), que habitualmente muestra un tumor polipoideo y pigmentado, en el tercio medio o el distal del esófago ${ }^{3}$. Sin embargo, también se pueden observar lesiones múltiples en $12 \%$ de los pacientes ${ }^{4}$. El diagnóstico se confirma con el estudio anatomopatológico que se debería apoyar en tinciones de inmunohistoquímica ${ }^{5}$.

En la mayoría de los estudios se ha utilizado la tomografía computada como medio estándar de etapificación ${ }^{3}$. En los últimos años, se ha descrito el rol de la tomografía de emisión de positrones con ${ }^{18} \mathrm{~F}$-deoxiglucosa $\left({ }^{18} \mathrm{FD}\right.$ PET-CT $)$, que permitiría una etapificación regional y sistémica más certera ${ }^{6}$.

Existen pocas comunicaciones en la literatura mundial sobre esta patología, generalmente series de pocos casos, que no permiten establecer comparaciones terapéuticas. Sin embargo, los mejores resultados a largo plazo se han observado en pacientes tratados con esofagectomía ${ }^{7}$. A pesar de ello, el pronóstico es malo, con una sobrevida global a 1 año de $30 \%$ y a 5 años de $5 \%{ }^{8}$.

El objetivo de este reporte es comunicar 2 nuevos casos de melanoma primario del esófago tratados con esofagectomía y discutir algunos aspectos de esta patología.

\section{Casos Clínicos}

Caso 1: Mujer de 51 años, domiciliada en Antofagasta, con antecedente de tabaquismo y asma. Consultó en junio de 2006 por disfagia 
lógica, náuseas, vómitos y baja de peso. La EDA mostró una lesión polipoidea, mamelonada y ulcerada en el tercio medio del esófago que comprometía casi todo el lumen esofágico. El estudio anatomopatológico de la lesión mostró un tumor sólido, infiltrante, con características morfológicas e inmunohistoquímicas compatibles con un melanoma maligno. La tomografía computada de tórax (Figura 1 A y B) mostró una lesión tumoral, con crecimiento intraluminal, en el tercio distal del esófago. La lesión comprometía el cardias y comprimía la aurícula izquierda. No hubo evidencia de compromiso de órganos adyacentes ni metástasis. La tomografía computada de abdomen $\mathrm{y}$ pelvis fue normal.

En julio de 2006, consultó en el Instituto Oncológico Fundación Arturo López Pérez (FALP). El examen físico y los exámenes generales preoperatorios fueron normales. Se le realizó

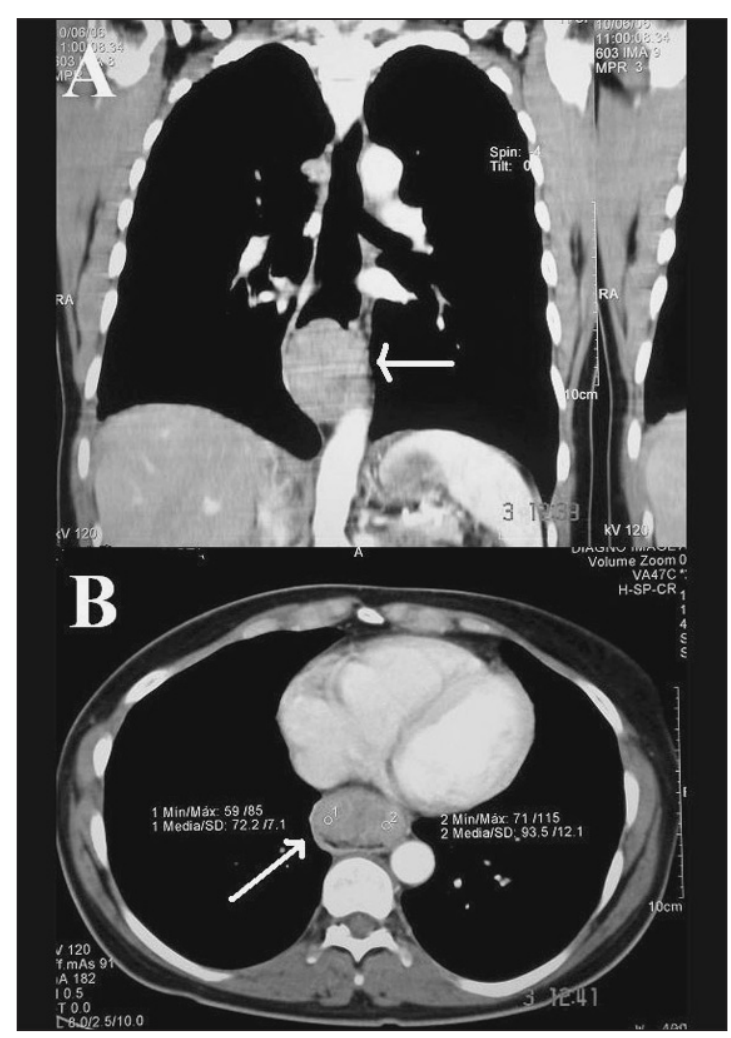

Figura 1. Tomografía Computada de Tórax. A: Corte coronal. Se observa una lesión polipoídea en el tercio del esófago (flecha blanca). B: Corte transversal. Se observa una lesión polipoídea que compromete todo el lumen del esófago distal (flecha blanca). una tomografía de emisión de positrones con ${ }^{18} \mathrm{~F}$-deoxiglucosa que mostró un tumor de $9 \mathrm{~cm}$ en el esófago distal y adenopatías hipercaptantes en el omento menor, con un índice de captación estandarizado promedio de glucosa (SUV) de 11,1 y $3,3 \mathrm{~g} / \mathrm{ml}$, respectivamente. Debido a la ausencia de metástasis a distancia se decidió la resección quirúrgica. El 20/7/2006 se le realizó una esofagectomía transhiatal, disección mediastínica en block, ascenso gástrico por mediastino posterior y anastomosis esofagogástrica cervical. La paciente evolucionó en forma satisfactoria y fue dada de alta, sin complicaciones, el día 12 postoperatorio. El estudio anatomopatológico mostró un melanoma melanocítico de $9 \mathrm{~cm}$ en el tercio inferior del esófago. La lesión tumoral comprometió hasta la túnica muscular propia. Además se observaron múltiples focos de melanoma in situ y permeaciones linfovasculares peritumorales. Los márgenes quirúrgicos fueron negativos, sin embargo, en el margen proximal se observaron algunos melanocitos atípicos aislados en el epitelio escamoso. Uno de 19 ganglios linfáticos resecados presentó compromiso tumoral por melanoma.

Cuatro meses después de la cirugía, debido a compromiso progresivo del estado general, se le realizó otra tomografía de emisión de positrones con ${ }^{18} \mathrm{~F}$-deoxiglucosa. En este examen se observaron al menos 7 lesiones compatibles con metástasis hepáticas (entre 10 y $75 \mathrm{~mm}$ de diámetro) junto a ganglios linfáticos de aspecto patológico, con aumento en la captación de glucosa en la axila izquierda. En todas las lesiones el SUV fluctuó entre 2,9 y 11,6 g/dl (Figura 2). La paciente falleció el 08/05/2007 como consecuencia de una progresión de la enfermedad.

Caso 2: Hombre de 59 años, domiciliado en Temuco, sin antecedentes mórbidos. Consultó en septiembre de 2007 por disfagia lógica asociada a baja de peso. La endoscopia digestiva alta demostró una lesión polipoidea de $5 \mathrm{~cm}$, pigmentada, en el tercio distal del esófago (Figura 3). El estudio anatomopatológico de la biopsia endoscópica diagnosticó un tumor maligno indiferenciado. En septiembre de 2007 fue evaluado en FALP. El examen físico y los exámenes de laboratorio fueron normales. El material anatomopatológico fue reevaluado y se concluyó que el tumor presentaba características morfológicas e inmunohistoquímicas concordantes con un melanoma maligno. 
Melanoma primario del esófago tratado con esofagectomía. Casos clínicos - J.M. Butte et al

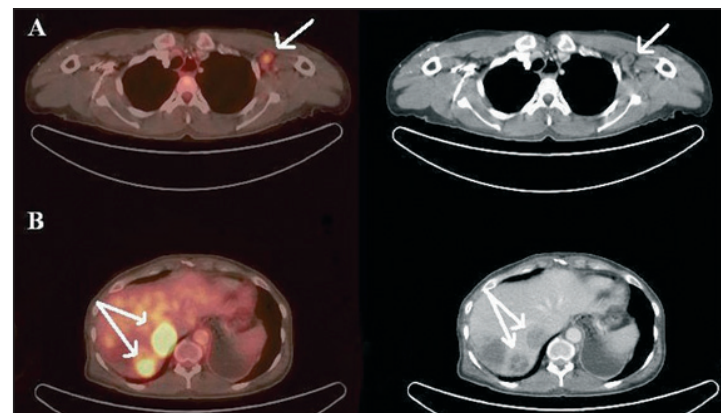

Figura 2. Tomografía de Emisión de Positrones con ${ }^{18} \mathrm{~F}$ deoxiglucosa. A: Lesión tumoral en axila izquierda con aumento de SUV (flecha). B: Lesiones metastásicas hepáticas con aumento de SUV (flechas).

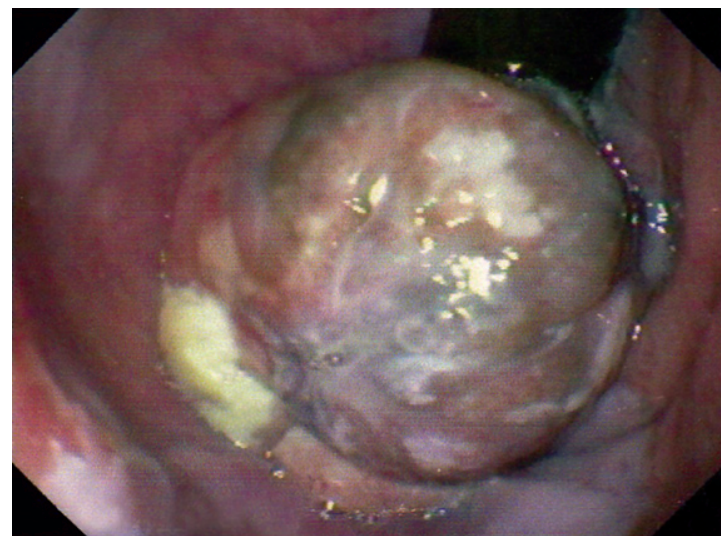

Figura 3. Endoscopia Digestiva Alta. Se observa una lesión polipoídea pigmentada en el extremo distal del esófago.



Figura 4. A (corte coronal) y B (corte transversal): Tomografía de emisión de positrones con ${ }^{18} \mathrm{~F}$-deoxiglucosa. Se observa una lesión tumoral de $9 \mathrm{~cm}$ en el tercio distal del esófago (flechas blancas), asociado a aumento del SUV.



Figura 5. Pieza quirúrgica de esofagectomía. A: Estudio macroscópico: Esófago abierto. En el extremo distal se observa una lesión polipoidea de $6 \mathrm{~cm}$ de eje mayor. B-Existe necrosis central y al corte hay compromiso de la capa muscular. C: Neoplasia maligna constituida por células grandes, con abundante citoplasma eosinófilo y núcleos con nucléolo prominente. (HE 400x). D: Estudio inmunohistoquímico para HMB-45 (positivo) en células tumorales. 
Una tomografía de emisión de positrones con ${ }^{18} \mathrm{~F}$-deoxiglucosa confirmó la presencia de una lesión polipoidea de $9 \mathrm{~cm}$, con un SUV máximo de 9,6 gr./ml, en el extremo distal del esófago. Además se observaron ganglios linfáticos peritumorales con aumento en la captación de glucosa hipercaptantes. No se identificaron metástasis a distancia (Figura 4). El 26/9/07 se le realizó una esofagectomía transhiatal, disección mediastínica en block, ascenso gástrico por mediastino posterior y anastomosis esofagogástrica cervical. El enfermo evolucionó en buenas condiciones y fue dado de alta, sin complicaciones, el día 11 postoperatorio. El estudio AP confirmó la presencia de un melanoma epiteloídeo, con alto índice mitótico, extensamente ulcerado, con infiltración inflamatoria del estroma, focalmente pigmentado y con necrosis central. El tumor infiltraba hasta la túnica muscular propia. Se observaron metástasis ganglionares en 3 de 10 ganglios linfáticos resecados. Los estudios inmunohistoquímicos para HMB-45 y la proteína S-100 fueron positivos (Figura 5).

En el último control de seguimiento (marzo de 2009), no hubo evidencias de recidiva clínica ni en las imágenes.

\section{Discusión}

En este estudio se describen dos pacientes tratados por un melanoma esofágico primario. Ambos tuvieron una presentación clínica, estudio preoperatorio y tratamiento quirúrgico similar, pero una diferente evolución y sobrevida. Al igual que la mayoría de los pacientes con esta patología, consultaron por disfagia y baja de peso y la endoscopia sugirió el diagnóstico de melanoma, al evidenciar una lesión polipoidea. Fue necesario utilizar tinciones de inmunohistoquímica para confirmar el diagnóstico anatomopatológico. Ambos fueron estudiados con una tomografía de emisión de positrones con ${ }^{18} \mathrm{~F}$-deoxiglucosa, que fue negativa para metástasis a distancia. Sin embargo, es lógico pensar que en la primera paciente fue falsamente negativa, ya que un nuevo examen realizado 4 meses después, mostró múltiples metástasis hepáticas. Estas tampoco se observaron en la exploración quirúrgica. La diferente evolución y sobrevida estaría fundamentalmente determinada por la presencia de enfermedad sistémica no diagnosticada al momento de la operación.
Los melanomas esofágicos primarios son tumores muy poco frecuentes. Hasta el año 2005 se habían descrito en la literatura mundial sólo 262 casos de melanoma primario del esófago 9 Cheung y cols ${ }^{10}$, en un estudio que abarcó 31 años (1973 a 2004), identificaron 659 pacientes con un melanoma primario del tracto gastrointestinal. No hubo predisposición por el sexo y $51 \%$ de los pacientes fue mayor de 70 años al momento del diagnóstico. La mayoría de los tumores se ubicaron en el canal anal o en la zona oro-nasal (boca, lengua, amígdalas o nasofaringe) y sólo en 41,2\% de los pacientes, la neoplasia estaba localizada. La sobrevida media de todos los casos fue de 17 meses y fue menor en aquellos con compromiso ganglionar, enfermedad diseminada o tratados en forma no quirúrgica. En este estudio sólo se describieron 39 pacientes $(5,9 \%)$ portadores de melanoma esofágico. También se observó que la sobrevida promedio de los enfermos con melanomas originados en el esófago fue de 12 meses y que aumentó luego del tratamiento quirúrgico (7 vs 12 meses en enfermos tratados en forma no quirúrgica y quirúrgica, respectivamente). Lamentablemente, esta información proviene de estudios retrospectivos que no permiten comparar diferentes tratamientos. Tampoco permiten establecer factores pronósticos independientes ni determinar si la sobrevida depende del tratamiento quirúrgico o de otros factores asociados.

Poco se conoce sobre la patogenia de esta enfermedad siendo difícil determinar si un melanoma del esófago corresponde a una metástasis visceral de un melanoma cutáneo o a un tumor primario. Varios estudios han asociado el desarrollo de un melanoma primario del esófago con la presencia de melanocitos en la unión del epitelio y el estroma esofágico (que se observa en el 2,5 a $8 \%$ de las personas sanas) y en la presencia de melanocitosis ${ }^{11}$. La melanocitosis corresponde al aumento del número de melanocitos en la capa basal del epitelio esofágico, asociado a una mayor cantidad de melanina en estos melanocitos ${ }^{11}$ y también ha sido descrita en asociación con esofagitis crónica, hiperplasia escamosa y carcinoma escamoso del esófago ${ }^{12}$. Chapman y cols ${ }^{13}$ describen que la hiperplasia melanocítica atípica y la presencia de focos de melanoma in situ serían signos histológicos distintivos del origen primario de estos tumores. En el estudio anatomopatológico de uno de los casos descritos, se observó la presencia de 
melanocitos atípicos y melanoma in situ, lo que apoyaría el diagnóstico de melanoma primario del esófago.

El diagnóstico preoperatorio de melanoma esofágico puede ser difícil de realizar. Es importante descartar en el examen clínico la presencia de un melanoma cutáneo o mucoso ${ }^{14}$ y las tinciones de inmunohistoquímica de la proteína S-100 ó HMB 45 permitirían diferenciar el diagnóstico del de un carcinoma escamoso mal diferenciado.

La utilidad de la tomografía de emisión de positrones $\operatorname{con}^{18} \mathrm{~F}$-deoxiglucosa en la etapificación regional y sistémica de las neoplasias primarias del esófago está bien sustentada. En el manejo del melanoma cutáneo metastásico ha permitido detectar con gran sensibilidad la presencia de lesiones viscerales ${ }^{15}$. En estos pacientes se utilizó este examen tanto en la etapificación preoperatoria como en la pesquisa de una eventual recurrencia. El melanoma esofágico tiene vías preferentes de diseminación a través del plexo submucoso y canales linfáticos hacia linfonodos regionales. Por ello, en la mayoría de los casos reportados con enfermedad localizada, el tratamiento quirúrgico resectivo mediante esofagectomía, ha sido la opción terapéutica inicial. Pese a ello, en la mayoría de las ocasiones la diseminación sistémica precoz es el hecho clínico que determina la pobre sobrevida. No hay consenso si ante la presencia de lesiones eventualmente pre malignas como la melanocitosis, la esofagectomía debiera ser el tratamiento recomendado.

El pronóstico del melanoma esofágico primario es pobre, con una sobrevida reportada de $30 \%$ al año y $5 \%$ a los 5 años. Existen sin embargo, reportes aislados de pacientes sometidos a esofagectomía que logran una sobrevida de 10 a 48\% a 5 años ${ }^{8,10}$.

\section{Referencias}

1. Kimura H, Kato H, Sohda M, Nakajima M, Fukai Y, Miyazaki $\mathrm{T}$, et al. Flat-type primary malignant melanoma of the esophagus treated by EMR: case report. Gastrointest Endosc 2005; 61: 787-89.

2. Chang F, Deere H. Esophageal melanocytosis morphologic features and review of the literature. Arch Pathol Lab Med 2006; 130: 552-57.
3. Stranks G, Mathai J, Rowe-Jones D. Primary malignant melanoma of the oesophagus: case report and review of surgical pathology. Gut 1991; 32: 828-30.

4. Joob A, Haines G 3Rd, Kies M, Shields T. Primary malignant melanoma of the esophagus. Ann Thorac Surg 1995; 60: 217-22.

5. Dicostanzo D, Urmacher C. Primary malignant melanoma of the esophagus. Am J Surg Pathol 1987; 11: 46-52.

6. Antoch G, Vogt F, Freudenberg L, Nazaradeh F, Goehde S, Barkhausen J, et al. Whole-body dual-modality PET/ $\mathrm{CT}$ and whole-body MRI for tumor staging in oncology. JAMA 2003; 290: 3199-206.

7. Ho K, Cheng J, Wee A, Soo K. Primary malignant melanoma of the esophagus with multiple esophageal lesions Nat Clin Pract Gastroenterol Hepatol 2007; 4: 171-4.

8. Sabanathan S, Eng J, Pradhan G. Primary malignant melanoma of the esophagus. Am J Gastroenterol 1989; 84: 1475-81.

9. Vandewoude M, Cornelis A, Wyndaele D, Brussaard C, Kums R. (18) FDG-PET-scan in staging of primary malignant melanoma of the oesophagus: a case report. Acta Gastroenterol Belg 2006; 69: 12-4.

10. Cheung M, Pérez E, Molina M, Jin X, Gutiérrez J, Franceschi D, et al. Defining the role of surgery for primary gastrointestinal tract melanoma. J Gastrointest Surg 2008; $12: 731-8$.

11. Oshiro T, Shimoji H, Matsuura F, Uchima N, Kinjo F, Nakayama T, et al. Primary malignant melanoma of the esophagus arising from a melanotic lesion: report of a case. Surg Today 2007; 37: 671-5.

12. Fredricks J, Bejarano P. Primary malignant melanoma of the esophagus with separate foci of melanoma in situ and atypical melanocytic hyperplasia in a patient positive for human immunodeficiency virus: a case report and review of the literature. Arch Pathol Lab Med 2008; 132: $1675-8$.

13. Chapman J, Bejarano P. Primary malignant melanoma of the esophagus with separate foci of melanoma in situ and atypical melanocytic hyperplasia in a patient positive for human immunodeficiency virus. Arch Pathol Lab Med 2008; 132: 1675-8.

14. Sánchez A, Wu T, Prieto V, Rashid A, Hamilton S, Wang $\mathrm{H}$. Comparision of primary and metastatic malignant melanoma of the esophagus. Arch Pathol Lab Med 2008; 132: 1623-9.

15. Maldonado A, González-Alenda F, Alonso M, Sierra J. PET-CT in clinical oncology Clin Transl Oncol 2007; 9: 494-505. 